\title{
Energy \& Environmental Science \\ High electrode activity of nanostructured, columnar ceria films for solid oxide fuel cells
}

\author{
WooChul Jung, Julien O. Dereux, William C. Chueh, † Yong Hao $\ddagger$ and Sossina M. Haile* \\ Received 4th May 2012, Accepted 20th July 2012 \\ DOI: 10.1039/c2ee22151a
}

\begin{abstract}
Highly porous oxide structures are of significant importance for a wide variety of applications in fuel cells, chemical sensors, and catalysis, due to their high surface-to-volume ratio, gas permeability, and possible unique chemical or catalytic properties. Here we fabricated and characterized $\mathrm{Sm}_{0.2} \mathrm{Ce}_{0.8} \mathrm{O}_{1.9-\delta}$ films with highly porous and vertically oriented morphology as a high performance solid oxide fuel cell anode as well as a model system for exploring the impact of electrode architecture on the electrochemical reaction impedance for hydrogen oxidation. Films are grown on single crystal YSZ substrates by means of pulsed laser deposition. Resulting structures are examined by SEM and BET, and are robust up to post-deposition processing temperatures as high as $900{ }^{\circ} \mathrm{C}$. Electrochemical properties are investigated by impedance spectroscopy under $\mathrm{H}_{2}-\mathrm{H}_{2} \mathrm{O}-\mathrm{Ar}$ atmospheres in the temperature regime $450-650{ }^{\circ} \mathrm{C}$. Quantitative connections between architecture and reaction impedance and the role of ceria nanostructuring for achieving enhanced electrode activity are presented. At $650{ }^{\circ} \mathrm{C}, p \mathrm{H}_{2} \mathrm{O}=0.02 \mathrm{~atm}$, and $p \mathrm{H}_{2}=0.98 \mathrm{~atm}$, the interfacial reaction resistance attains an unprecedented value of 0.21 to $0.23 \Omega \mathrm{cm}^{2}$ for porous films $4.40 \mu \mathrm{m}$ in thickness.
\end{abstract}

\section{Introduction}

Due to their combination of high efficiency and fuel flexibility, solid oxide fuel cells (SOFCs) can play a critical role in a carbonneutral, sustainable energy future. ${ }^{1}$ A key step in the fuel-cell energy conversion process is hydrogen electro-oxidation, $\mathrm{H}_{2}$

Materials Science, California Institute of Technology, Pasadena, CA 91125, USA. E-mail: smhaile@caltech.edu; Fax: +1 626-395-8868; Tel: +1 626-395-2958

$\uparrow$ Current address: Materials Science and Engineering, Stanford University, Stanford, CA 94305, USA.

\$ Current address: Institute of Engineering Thermophysics, Chinese Academy of Sciences, Beijing 100190, P. R. China. (gas) $+\mathrm{O}^{2-} \rightarrow \mathrm{H}_{2} \mathrm{O}$ (gas) $+2 \mathrm{e}^{-}$, at the anode. State-of-the-art SOFCs typically employ porous composite anodes formed of an ionically conducting oxide and a catalytically active, electronically conducting metal, most commonly $\mathrm{Ni}^{2}{ }^{2}$ The hydrogen electro-oxidation reaction is generally assumed to take place only within a limited region near the three-phase boundaries (3PBs), where the electronic conductor, ion conductor, and gas phase are in simultaneous contact. ${ }^{2}$ The conventional view further holds that the metal component provides the site for catalytic activity. In composite anodes in which the traditional YSZ (yttria stabilized zirconia) is replaced with an oxide of substantial electronic conductivity (in addition to high ionic conductivity) and high surface catalytic activity, however, the electrochemically active

\section{Broader context}

Solid oxide fuel cells (SOFCs) are expected to play a critical role in a carbon-neutral, sustainable energy future given their combination of high efficiency and fuel flexibility. One of the key steps in the fuel-cell energy conversion process is hydrogen electrooxidation, $\mathrm{H}_{2}$ (gas) $+\mathrm{O}^{2-} \rightarrow \mathrm{H}_{2} \mathrm{O}$ (gas) $+2 \mathrm{e}^{-}$, at the anode. Despite extensive research efforts to achieve high reaction rates by engineering anode microstructures, results have remained unsatisfactory. Limited understanding of the connections between the electrode architecture and the hydrogen electro-oxidation reaction rate has slowed progress in this arena. Here we employ pulsed laser deposition (PLD) as a route to create $\mathrm{Sm}_{0.2} \mathrm{Ce}_{0.8} \mathrm{O}_{1.9-\delta}$ films with highly porous and vertically oriented morphology. In contrast to typical anodes, composites formed from yttria-stabilized zirconia (YSZ) as the ion transport component and nickel as the electron transport component, ceria based anodes provide multi-functionality in the single oxide component. Furthermore, the PLD synthesized films have an ideal morphology for presenting high specific surface area yet high rates of gas-phase mass transport (i.e., low tortuosity). As such, they not only enable quantitative connections between architectural features and electrochemical

behaviors, but ultimately yield anodes of unprecedented activity. 
region for hydrogen electro-oxidation can be expected to extend beyond the 3PBs to the two-phase boundary (2PB) between the oxide and gas interface. Such behavior has, in fact, recently been demonstrated by the present authors in the case of doped ceria. ${ }^{3}$ Expansion of the active zone to the entire area of 2PBs in a porous electrode can, in turn, be anticipated to enhance the overall electrode reaction rate over that in a $3 \mathrm{~PB}$ dominated process. ${ }^{4-7}$

To take full advantage of the $2 \mathrm{~PB}$ reaction pathway in highactivity, mixed electron and oxygen ion conducting oxides, porous structures with high specific area, well-defined features, and high thermal stability are desirable. Typical fabrication routes, however, yield random structures that must be described by multiple microstructural parameters including porosity, pore size distribution, tortuosity, specific surface area, and percolation characteristics of the ion and electron transport pathways. Consequently, prediction and ultimate optimization of the activity of typical porous structures is extremely challenging. ${ }^{8-10}$ Moreover, insufficient specific surface area can necessitate very high temperature operation for satisfactory electrode performance, during which structures suffer from thermally induced coarsening, degrading electrode activity. To address these challenges, we employ pulsed laser deposition as a route to fabricate porous films with reproducible and controllable features. We show that not only are films of $\mathrm{Sm}_{0.2} \mathrm{Ce}_{0.8} \mathrm{O}_{1.9-\delta}$ (SDC, a material of mixed electron and oxygen ion conductivity under reducing conditions) prepared by this route thermally stable, but also that they enable quantitative connections between architectural features and electrochemical properties and ultimately yield SOFC anode structures of unprecedented activity.

\section{Experimental procedures}

Electrochemical cells with a symmetric geometry (electrode|electrolyte|electrode) were fabricated using $10 \times 10 \times$ $0.5 \mathrm{~mm}$ (100) $\mathrm{Y}_{0.16} \mathrm{Zr}_{0.84} \mathrm{O}_{1.92}$ (MTI Corp.) single crystals as the oxygen-ion-conducting solid electrolyte. The $\mathrm{Sm}_{0.2} \mathrm{Ce}_{0.8} \mathrm{O}_{1.9-\delta}$ (SDC) electrodes were grown via pulsed-laser deposition (PLD, Nocera, with Coherent $102 \mathrm{KrF} 248 \mathrm{~nm}$ excimer laser, $\sim 2 \mathrm{~J} \mathrm{~cm}^{-2}, 20$ $\mathrm{Hz}$ ) on both sides of the YSZ electrolyte. The PLD targets were prepared from high purity commercial SDC (Nextech Materials). Powders were compacted at $350 \mathrm{MPa}$ using an isostatic press and then sintered at $1250^{\circ} \mathrm{C}$ for $5 \mathrm{~h}$. To avoid contamination during film growth, sacrificial layers, also YSZ, were placed between the substrate and the PLD heater stage. The growth temperature, measured by a thermocouple in direct contact with the YSZ wafer (bonded with $\mathrm{Pt}$ adhesive paste), was $\sim 650^{\circ} \mathrm{C}$. Average heating rates were $10^{\circ} \mathrm{C} \mathrm{min}^{-1}$. The deposition temperature and atmosphere were $650{ }^{\circ} \mathrm{C}$ and $100 \mathrm{mTorr} \mathrm{O}_{2}$, respectively, conditions found to yield porous rather than dense films. Film thickness was varied between 0.22 and $10.8 \mu \mathrm{m}$ by varying deposition time from 8 to $416 \mathrm{~min}$.

The microstructure was examined using a Carl Zeiss LEO 1550VP scanning electron microscope (SEM), and analyzed by ImageJ (National Institute of Health) software. Cross-sectional images were obtained from samples milled by focused ion beam methods (FEI Helios Nanolab, a $30 \mathrm{kV}, 0.92 \mathrm{nA}$ ) after vacuuminfiltration with a low-viscosity resin (Buehler Epothin). In this case SEM images were collected using a ZEISS Ultra 55 scanning electron microscope. X-ray diffraction (XRD) was used to determine the crystal structure, phase purity, and orientation distributions of the films using a Philips X'Pert Pro MRD Pro diffractometer ( $\mathrm{Cu} \mathrm{K} \alpha$ source, $45 \mathrm{kV}, 40 \mathrm{~mA}$, 4-crystal Ge(220)/ (440) monochromator), equipped with a four-circle goniometer. In-plane X-ray diffraction patterns ( $\phi$ scan) were collected for the (111) peak after tilting the specimen by $54.7^{\circ}$. BET measurements (Micrometritics ASAP 2020) were performed to assess specific surface area of selected samples, specifically, films grown to 1.08 and $4.40 \mu \mathrm{m}$ in thickness. In order to ensure sensitivity of the technique (which requires a large total surface area), eight identical symmetric samples with SDC films on each side of the respective YSZ substrate were prepared and measured simultaneously using $\mathrm{Kr}$ gas at $77 \mathrm{~K}$.

The electrochemical characteristics of the porous films were measured by A.C. impedance spectroscopy (ACIS, Solartron 1260 , perturbation voltage of 20 to $40 \mathrm{mV}$ ), carried out under controlled temperatures and atmospheres under zero bias using a geometrically symmetric configuration. Both $\mathrm{Pt}$, typically considered highly active for hydrogen electro-oxidation, and $\mathrm{Au}$, considered catalytically inert (but more difficult to apply), were used as current collectors. Pastes of the respective metals were applied to the porous SDC electrode surfaces by brush-painting over a silk-screening mask. The Pt paste (Engelhard 6082) was subsequently fired at $900{ }^{\circ} \mathrm{C}$ for two h, whereas the Au paste (Fuelcellmaterials Au-I-10) was fired at $700{ }^{\circ} \mathrm{C}$ for two h. In the case of the $\mathrm{Au}, \mathrm{a} 100 \mathrm{~nm} \mathrm{Au}$ layer was deposited by sputtering (AJA International) before applying the paste, so as to improve contact with the current collector. The cells were placed inside a continuous-flow alumina tube to which $\mathrm{H}_{2}-\mathrm{H}_{2} \mathrm{O}-\mathrm{Ar}$ gas mixtures were delivered via digital mass flow controllers, with some of the gas, as required to attain the target $p \mathrm{H}_{2} \mathrm{O}$, passing through a water bubbler placed inside an isothermal water bath. The total flow

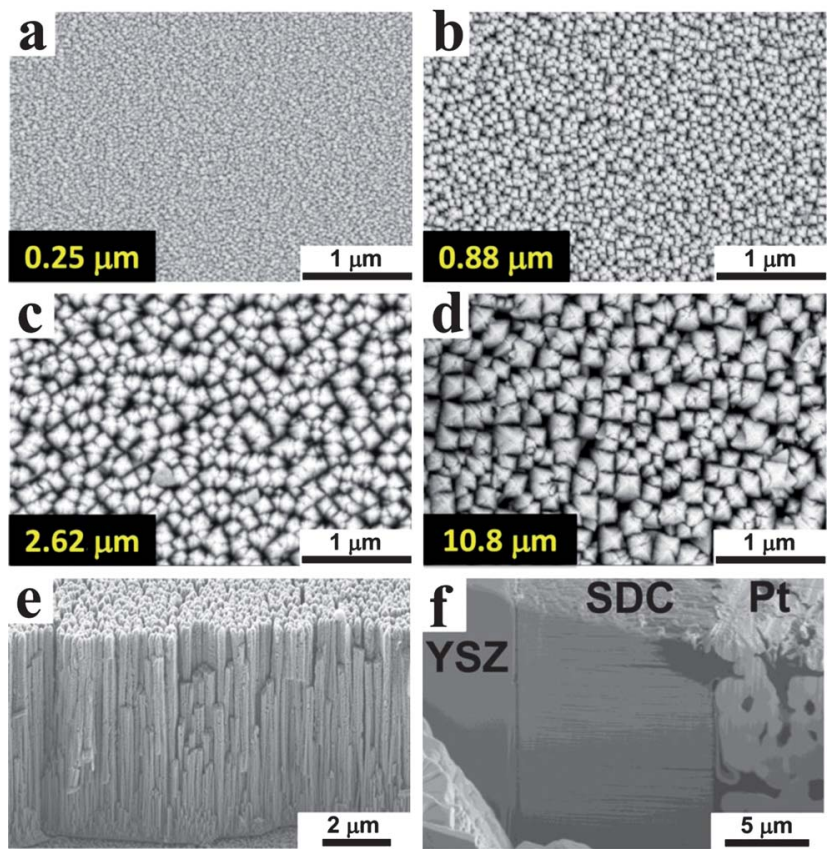

Fig. 1 Scanning electrode microscope micrographs of the porous columnar SDC films prepared in $100 \mathrm{mTorr}_{2}$ at $650^{\circ} \mathrm{C}$. (a-d) Top view image as a function of the layer thickness $(250 \mathrm{~nm}$ to $10.8 \mu \mathrm{m})$. (e-f) Cross-section view images with the Pt current collector on top. Image (f) was obtained after focused ion beam (FIB) milling of sample. 

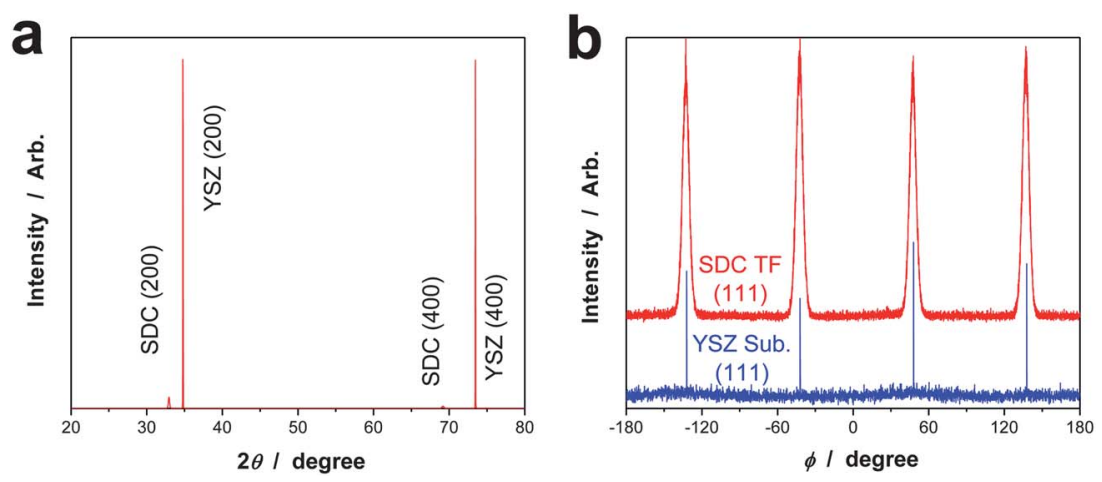

Fig. 2 X-ray diffraction data for porous columnar SDC thin film, $2.32 \mu \mathrm{m}$ in thickness, grown onto a single crystal (100) YSZ substrate. (a) In-plane diffraction pattern ( $2 \theta$ scan), and (b) out-of-plane diffraction patterns ( $\phi$ scan) comparing the YSZ substrate and the SDC deposited sample. Each $\phi$ scan was collected for the (111) peak after tilting the sample by $54.7^{\circ}$.

rate was kept constant at $101 \mathrm{~cm}^{3} \mathrm{~min}^{-1}$ (standard temperature and pressure) and both sides of the cell exposed to the single gas environment. Effluent humidity was monitored using a Rotronic Hygroflex capacitive sensor. Data were analyzed using ZView software (Scribner Associates, version 2.9b). Area-normalized resistance and capacitance values are designated with a $\sim$, where $\tilde{R}$ is $R \times A, \tilde{C}$ is $C / A$ and $A$ is the projected electrolyte area.

\section{Results and discussion}

SEM micrographs of representative SDC films are shown in Fig. 1. The films display well-defined, high surface-area morphologies, as desired. The oxide grows in the form of vertically aligned square columns under the optimized pressure of

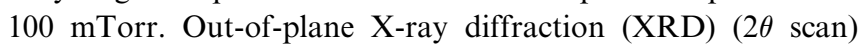
showed only (100) cubic fluorite peaks, indicative of highly oriented texture (Fig. 2a). The number and position of diffraction peaks observed by in-plane XRD ( $\phi$ scan) was also consistent with the rotational symmetry of the crystallographic plane normal to the incident beam, indicating alignment registry between the film and the substrate (Fig. 2b). Deposition under lower oxygen pressures (a few mTorr) led to dense films, ${ }^{3,11}$ whereas higher pressures ( $\sim 200 \mathrm{mTorr}$ ) led to random, disordered microstructures (not shown). The vertical alignment of both the pores and the oxide features indicates that diffusion of electronic, ionic, and gas phase species will be largely confined to one dimension, providing the desired simplification of the complex electrochemical reaction pathways.

The images in Fig. 1 reveal that the SDC columns thicken as they grow and, simultaneously, their number density (i.e., number of columns per unit projected area) decreases. Quantitative evaluations of these and additional relevant microstructural features - column width, column number density, surface area enhancement factor, and specific area - and their dependences on film thickness are provided in Fig. 3. The column width is defined as the average diagonal distance across the top of the columns (evaluated for 90 to 140 columns per sample), whereas the number density of columns was determined from an evaluation of the area that encompassed $\sim 100$ columns (ranging from 0.2 to $2 \mu \mathrm{m}^{2}$ area of film). The former increases from 46.1 $\mathrm{nm}$ for the shortest columns to $249.8 \mathrm{~nm}$ for the tallest, whereas the latter decreases from $6.56 \times 10^{10} \mathrm{~cm}^{-2}$ to $2.14 \times 10^{9} \mathrm{~cm}^{-2}$ (Fig. 3a), and both quantities display a power law dependence on column height.

From the apparent power law dependences of the measured microstructural features (Fig. 3a) and an assumption of smooth, slightly conical, cylindrical columns, the normalized total ceria surface area of the films, $S_{\text {total }}$ (total area per projected area), was estimated as a function of film thickness (or column height), $H$, according to a

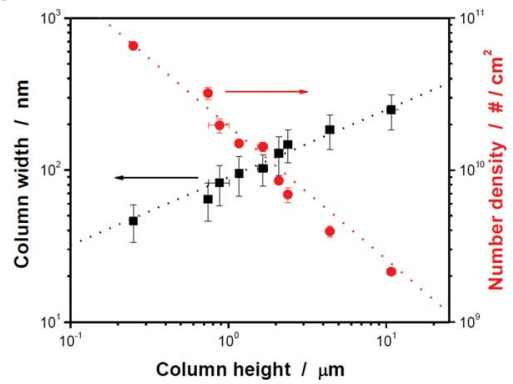

b

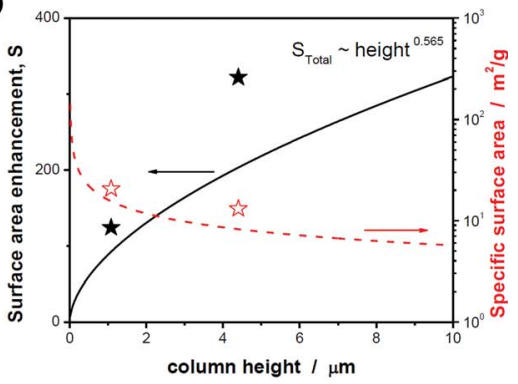

Fig. 3 (a) Double-logarithmic plots of the column width (left axis) and number density (right axis) as functions of column height (or film thickness), and (b) surface area enhancement (left axis) and specific (gravimetric) surface area (right axis) as functions of column height (or film thickness) as expressed in eqn (1) and (2), respectively. Error bars in (a) represent the distribution in values obtained from the analyses. In (b) data obtained directly from BET measurements for the $1.08 \mu \mathrm{m}$ and the $4.40 \mu \mathrm{m}$ thick films are also presented for comparison (stars). 


$$
S_{\text {total }} \approx \int_{0}^{H} P(h) \times N(h) \mathrm{d} h \approx 88.52 \times H^{0.565}
$$

where $P(h)$ is the average column perimeter at a given film height and $N(h)$ is the number density of columns at a given film height. As $S_{\text {total }}$ is normalized to the projected surface area, it is identical to the surface area enhancement factor (Fig. 3b). In analogy to the surface area, the volume of ceria deposited per projected area is approximated as

$$
V_{\text {total }} \approx \int_{0}^{H} A(h) \times N(h) \mathrm{d} h \approx 7.72 \times 10^{-5} \times H^{1.01}
$$

where $A(h)$ is the average column cross-sectional area at a given film height. This second expression, in combination with the material density of $7.15 \mathrm{~g} \mathrm{~cm}^{-3}$ permits estimation of the conventional specific surface area (area per mass) (Fig. 3b). It also enabled an estimate of the porosity, a quantity discussed below.

The various approximations not-with-standing, both the BET and microstructural investigations reveal that the surface area enhancement factors and specific areas of the films are enormous. From the direct BET measurements, these quantities are, respectively, $124 \pm 1$ and $20.6 \pm 0.1 \mathrm{~m}^{2} \mathrm{~g}^{-1}$ for the $1.08 \mu \mathrm{m}$ thick film, and $322 \pm 2$ and $13.1 \pm 0.1 \mathrm{~m}^{2} \mathrm{~g}^{-1}$ for the $4.40 \mu \mathrm{m}$ thick film. In the case of the thicker film, the surface enhancement factor exceeds that encountered in porous anodes prepared by conventional powder processing (screen-printing and subsequent partial sintering 6,10,12) by more than an order of magnitude. The specific surface areas are in fact typical of oxide powders with sub-micron-sized particles. ${ }^{10}$ The microstructurally derived values, though also extremely high, are somewhat lower than those from the direct measurements. This difference (of 34 and $58 \%$ in surface area enhancement, respectively, for the two films) is likely a result of nanoscale roughness, as evident, for example, in Fig. 1c, which is not captured in the analytical formalism employed.

Beyond the overall magnitudes of the specific surface area and surface area enhancement factor, there are two other relevant findings regarding the columnar structure. First, while the total surface area increases with film thickness, it does so non-linearly. The microstructural analysis suggests a power-law dependence of area $\propto$ thickness $^{0.565}$, whereas the limited BET data set suggest a slighlty stronger dependence on thickness (an exponent of $\sim 0.7$ ). Accordingly, any process that scales with surface area would not be expected to directly scale with film thickness. Second, from Fig. 1f, it is evident that the large-grained $\mathrm{Au}$ and $\mathrm{Pt}$, which were used as current collectors on top of the SDC films, remain confined to the exterior surface of the structure and do not penetrate into the nanoscale porosity. Therefore, contributions to the electrochemical activity from these species are not expected.

Selected results of the electrochemical impedance measurements are provided in Fig. 4. The raw impedance spectra, examples of which are presented in Fig. 4a, generally exhibited an
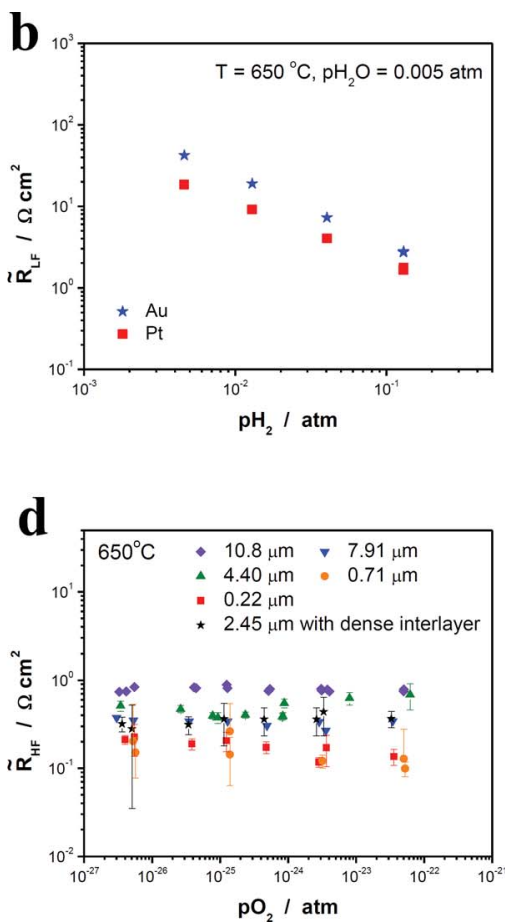

Fig. 4 (a) Typical impedance spectra obtained from Au|SDC|YSZ(100)|SDC|Au (stars) and Pt|SDC|YSZ(100)|SDC|Pt (squares) symmetric cells at $T=$ $650^{\circ} \mathrm{C}, p \mathrm{H}_{2}=0.10 \mathrm{~atm}$ and $p \mathrm{H}_{2} \mathrm{O}=0.005 \mathrm{~atm}$. The SDC layers are $2.55 \mu \mathrm{m}$ thick for both cases. (b) Double-logarithmic plot of the LF electrode resistance vs. $p \mathrm{H}_{2}$ measured for the same samples at $T=650^{\circ} \mathrm{C}$ and $p \mathrm{H}_{2} \mathrm{O}=0.005 \mathrm{~atm}$. (c) Arrhenius plot of the LF electrode resistance $v s$. temperature measured for the same samples under $p \mathrm{H}_{2}$ of 0.98 atm and $p \mathrm{H}_{2} \mathrm{O}$ of $0.02 \mathrm{~atm}$. (d) Double-logarithmic plots of the $\mathrm{HF}$ electrode resistance $v s$. $p \mathrm{O}_{2}$ measured for samples with different SDC film thicknesses at $650{ }^{\circ} \mathrm{C}$. (Star) a $250 \mathrm{~nm}$ thick, dense SDC interlayer was deposited onto a YSZ substrate before subsequently depositing a $2.45 \mu \mathrm{m}$ thick, porous SDC layer. $\tilde{R}$ represents resistance values normalized by the projected electrode area $(\tilde{R}=R \times$ Area $)$. 

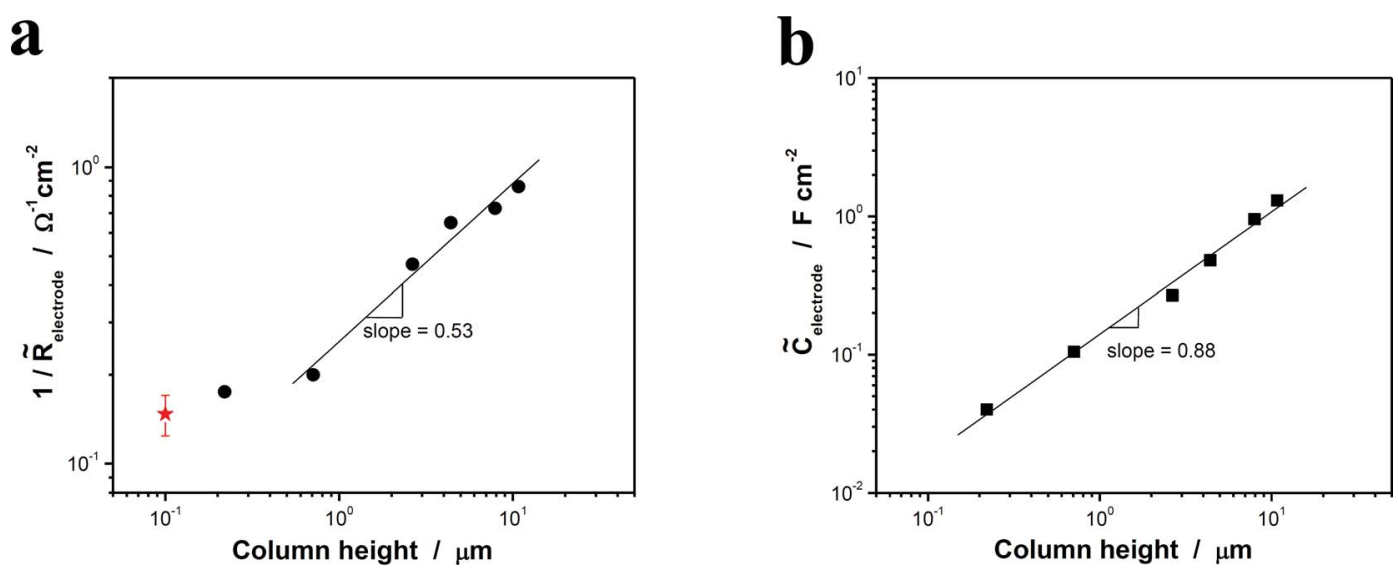

Fig. 5 (a) Variation in electrode activity (defined as 1/electrode resistance, as normalized to the projected electrode area) with column height, at $T=650^{\circ} \mathrm{C}$, $p \mathrm{H}_{2}=0.10 \mathrm{~atm}, p \mathrm{H}_{2} \mathrm{O}=0.050$ atm and $p \mathrm{O}_{2}$ of $1.3 \times 10^{-25}$ atm. For comparison, the electrode activity obtained from a dense and flat SDC film (ref. 3 ) is also presented (star), placed at an arbitrary value of column height. (b) Variation in electrode capacitance, as normalized to the projected electrode area, with column height under the same measurement condition. $\tilde{C}$ represents capacitance values normalized by the projected electrode area $(\tilde{C}=C / A r e a)$

offset resistance $\left(R_{\text {off }}\right)$ at high frequency, evident as a displacement of the data along the real axis, and an ideal or nearly ideal semicircle at lower frequency. In some cases, a small, distorted semicircle was additionally found at higher frequency, but only when Pt was utilized as a current collector (Fig. 4a insert). In a previous study we showed unequivocally, using dense SDC films and patterned current collectors, that the resistance associated with the nearly ideal arc is due to the surface electrochemical reaction resistance, whereas the offset is due to the bulk resistance of the YSZ electrolyte. ${ }^{3}$ The spectra are interpreted similarly here, as supported in detail below. The assignment of the offset resistance to the electrolyte response is explicitly justified by the observation here that $R_{\text {off }}$ was independent of gas phase composition and electrode thickness and displayed an activation energy $(\sim 0.9 \mathrm{eV})$ and overall magnitude (implying a conductivity of $5 \times 10^{-3} \mathrm{~S} \mathrm{~cm}^{-1}$ ) that are typical of ionic conductivity in YSZ (i.e., reported apparent activation energy of $1.1 \mathrm{eV}$ and conductivity of $1 \times 10^{-2} \mathrm{~S} \mathrm{~cm}^{-1}$ at $650^{\circ} \mathrm{C}$ (ref. 13)).

Turning to the prominent, nearly ideal arc in each of the spectra in Fig. 4a, the capacitance implied for this response is $\sim 0.3 \mathrm{~F} \mathrm{~cm}^{-2}$, irrespective of current collector metal. This large capacitance greatly exceeds typical interfacial values for electrochemical reactions at planar or low roughness surfaces, generally on the order of $\sim 10^{-3} \mathrm{~F} \mathrm{~cm}^{-2}{ }^{9,14}$ The assignment of the arc to the response of the ceria film is, in fact, consistent with an unusually large capacitance; the two contributing terms, the bulk chemical capacitance of the mixed conducting ceria and the interfacial capacitance of the ceria-gas interface are each large, the former because of the variable ceria stoichiometry and the latter because of the very high surface area. The resistance associated with this arc is then attributed specifically to the electrochemical reaction resistance for the oxidation of hydrogen at the ceria-gas interface. Such an interpretation, which is analogous to that presented for dense films, ${ }^{3,11}$ is further supported by the observation that the resistance measured here is, to first order, independent of the choice of current collector, Au or Pt. The temperature and gas pressure dependences are identical (Fig. $4 \mathrm{~b}$ and c), and the approximately $40 \%$ higher resistance in the case of Au (applied by sputter-deposition) may plausibly reflect a greater coverage of the ceria, blocking gas phase access to catalytic sites. In any case, it is evident that the sputtered $\mathrm{Au}$ layer has no beneficial impact on the resistance of this response. Electronic and/or ionic charge transport along the columns are readily eliminated as possible rate-limiting-steps because of the highly symmetric shape of the impedance arc. Furthermore, the area-specific resistances for ionic and electronic transport across a distance of $\sim 20 \mu \mathrm{m}$ (based on reported conductivities ${ }^{14}$ ) are $0.07 \Omega \mathrm{cm}^{2}$ and 0.016 to $0.04 \Omega \mathrm{cm}^{2}$, respectively, at the temperature $\left(650^{\circ} \mathrm{C}\right)$ and atmosphere conditions considered, far lower than the measured impedance values of 2 to $20 \Omega \mathrm{cm}^{2}$. Finally, gas-phase mass transport is eliminated on the basis of measurements demonstrating independence of the arc features to total gas flow rate over the range 50 to $100 \mathrm{~cm}^{3} \mathrm{~min}^{-1}$ (data not shown), indicating that even at half the flow rate used in the measurements, mass transport limitations are negligible.

In contrast to the symmetric, low frequency arc, the distorted, higher frequency arc was found to display a resistance independent of oxygen partial pressure and a magnitude that increased with film thickness (Fig. 4d). Furthermore, as noted above, this feature was generally absent when utilizing $\mathrm{Au}$ as a current collector. On the other hand, its characteristics were unchanged by the introduction of a very thin dense interlayer of SDC

Table 1 Detailed physical and electrochemical parameters of the SDC films for Fig. 5. Both electrode resistance and capacitance were obtained under $p \mathrm{H}_{2}$ of $0.10 \mathrm{~atm}, p \mathrm{H}_{2} \mathrm{O}$ of $0.005 \mathrm{~atm}$ and $p \mathrm{O}_{2}$ of $1.3 \times 10^{-25}$ atm at $650{ }^{\circ} \mathrm{C}$

\begin{tabular}{llll}
\hline $\begin{array}{l}\text { Thickness } \\
\mu \mathrm{m}\end{array}$ & $\begin{array}{l}\text { Surface area } \\
\text { enhancement, } S\end{array}$ & $\begin{array}{l}\text { Electrode } \\
\text { resistance, } \\
R_{\text {electrode }} \Omega \mathrm{cm}^{2}\end{array}$ & $\begin{array}{l}\text { Electrode } \\
\text { capacitance, } \\
C_{\text {electrode }} \mathrm{F} \mathrm{cm}\end{array}$ \\
\hline 0.22 & 38.1 & 5.73 & 0.04 \\
0.71 & 73.1 & 5.01 & 0.11 \\
2.65 & 152.9 & 2.13 & 0.27 \\
4.40 & $203.4\left(332^{a}\right)$ & 1.54 & 0.48 \\
7.91 & 283.1 & 1.38 & 0.95 \\
10.78 & 337.1 & 1.16 & 1.30
\end{tabular}

${ }^{a}$ Direct BET measurement. 

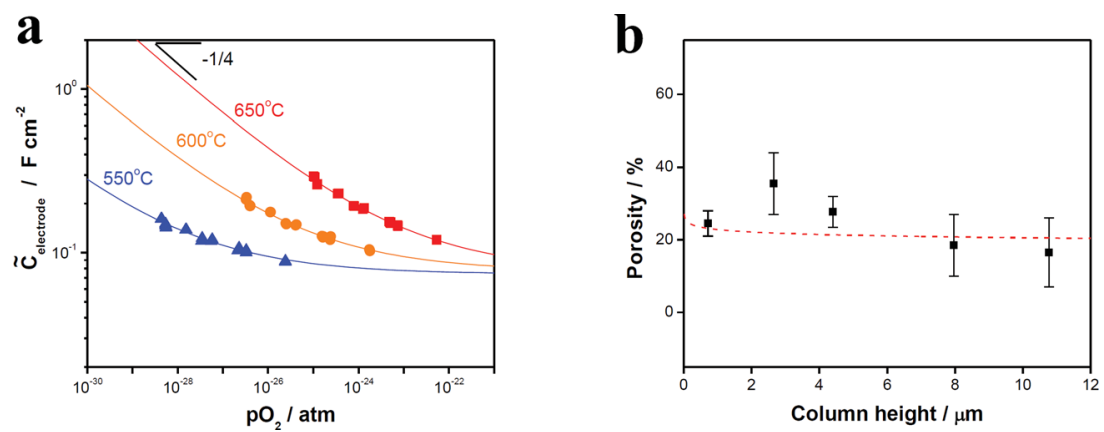

Fig. 6 (a) Double-logarithmic plots of the capacitance $v$ s. $p \mathrm{O}_{2}$ measured between 550 and $650{ }^{\circ} \mathrm{C}$ for a $2.65 \mu \mathrm{m}$ thick SDC film. Solid lines are fits to the expression $\tilde{C}=\tilde{B} \times p \mathrm{O}_{2}^{-1 / 4}+\tilde{D}$. (b) Porosity as a function of column height estimated from (square) the capacitance analysis and (dashed line) the $\mathrm{SEM}$ image analysis.

between the YSZ substrate and the SDC columnar film. Accordingly, the response is preliminarily attributed to poor contact between the metal and the porous oxide, where the roughness of the latter increases with film thickness. As this response does not appear to be inherent to the behavior of the films (not only does sputtered Au eliminate it, alternative current collection geometries with superior contact characteristics can also be conceived), it is not considered further in this work.

With the physical origins of the impedance behavior identified, the electrochemical reaction activities of the porous SDC films for hydrogen electro-oxidation (associated with the symmetric, low-frequency arc) were evaluated, where activity is defined here to be 1/electrode resistivity (as normalized to the electrolyte projected area). The impedance behavior was well-represented by an $R Q$ sub-circuit, where the $n$-value in the constant phase element $(Q)$ with impedance $Z=\frac{1}{Y(i \omega)^{n}}$, where $Y$ is a constant and $\omega$ is frequency, fell within the range 0.94 to 0.98 . The dependence of activity on column height (film thickness) is presented in Fig. 5 and detailed in Table 1 for a given set of temperature and atmosphere conditions. For completeness, the capacitance behavior is also presented. The electrode activity clearly rises with increasing thickness, showing a five-fold increase over the range of film thicknesses explored. Furthermore, the dependence on film thickness is clearly non-linear. Including all data points, the slope in the double-logarithimic plot of activity versus column height is $0.45 \pm 0.05$, and excluding the first, an outlier, the slope is $0.53 \pm 0.05$. Such behavior is expected for a process which depends on surface area and where the latter, in turn, displays a non-linear dependence on column height. Indeed, the power law behavior observed here of activity $\propto$ thickness $^{n}$ is almost identical to the power law dependence of surface on thickness, which displays an exponent of 0.565 based on the SEM image analysis. The direct BET measurements also indicate a process that scales with surface area. Interpolating a surface activity of $0.26 \Omega^{-1} \mathrm{~cm}^{-2}$ for the $1.08 \mu \mathrm{m}$ thick film, we find that the ratio between activity and surface area enhancement factor $(0.26: 124)$ of this film is almost identical to that of the $4.40 \mu \mathrm{m}$ thick film $(0.65: 332)$. These data provide additional evidence that the impedance-governing process is the electrochemical reaction at the SDC|gas interface. The result further indicates that even the $10.8 \mu \mathrm{m}$ thick electrode is almost uniformly active in terms of the hydrogen electro-oxidation reaction. In turn, activity throughout the $10.8 \mu \mathrm{m}$ film implies that electronic and ionic diffusion lengths are short relative to its volumetric specific surface area of $\sim 3 \times 10^{5} \mathrm{~cm}^{2} \mathrm{~cm}^{-3}$ such that neither mass nor charge transport is rate limiting, as already deduced from the symmetry of the impedance arc. It is noteworthy that, because the current collector is applied atop the porous ceria film and does not make uniform contact to all of the columns (Fig. 1f), the electron migration distance can be greater than even twice the film thickness, and, nevertheless, electronic resistance does not limit the reaction rate.

The dependence of electrode activity on specific surface area observed here is much higher than one would obtain from an infinitely thick electrode (i.e., thickness greatly exceeds the extent of the active region), which is microstructurally modified to vary its volume specific surface area. In particular, it has been shown in a theoretical analysis that for such thick electrodes the activity will be proportional to the square root of the specific surface area. ${ }^{15}$ Thus, a 10 times improvement in the electrode activity of an infinitely thick electrode would require a 100 times increase in surface area. Here, enhancement in activity is achieved simply by increasing the film thickness because the entire film remains active even at a thickness of almost $11 \mu \mathrm{m}$.

Turning to an analysis of the capacitance, because chemical capacitance scales with material volume (without contributions from pores), ${ }^{11}$ this property can, in principle, be used to obtain an estimate of the film density. However, the measured capacitance is expected to have significant additional interfacial contributions as a result of the large SDClgas surface area. Indeed, as evident in Table 1, not only is the capacitance for these films large, it generally increases with film thickness, as would be anticipated for such effects. Decomposition of the measured capacitance into the two contributing terms was carried out by noting that the chemical capacitance depends on $p \mathrm{O}_{2}$ through its dependence on electronic charge carrier concentration, whereas the interfacial contributions have been found to be independent of $\mathrm{pO}_{2}{ }^{11}$ Specifically the total capacitance was evaluated as a function of oxygen partial pressure for each film and used to fit the expression $C=B \times p \mathrm{O}_{2}^{-1 / 4}+D$, where the first term represents the bulk chemical capacitance ( $C_{\text {chem }} \approx \frac{e^{2} A L}{k_{\mathrm{B}} T} c_{\text {eon }}$ where $e$ and $k_{\mathrm{B}}$ have their usual meanings, and $A, L$, and $c_{\text {eon }}$, are the sample cross-sectional area, thickness (or equivalently height, $H$ ) and electron concentration, respectively), and the second the interfacial capacitance. An example of the analysis methodology is presented in Fig. 6a for 

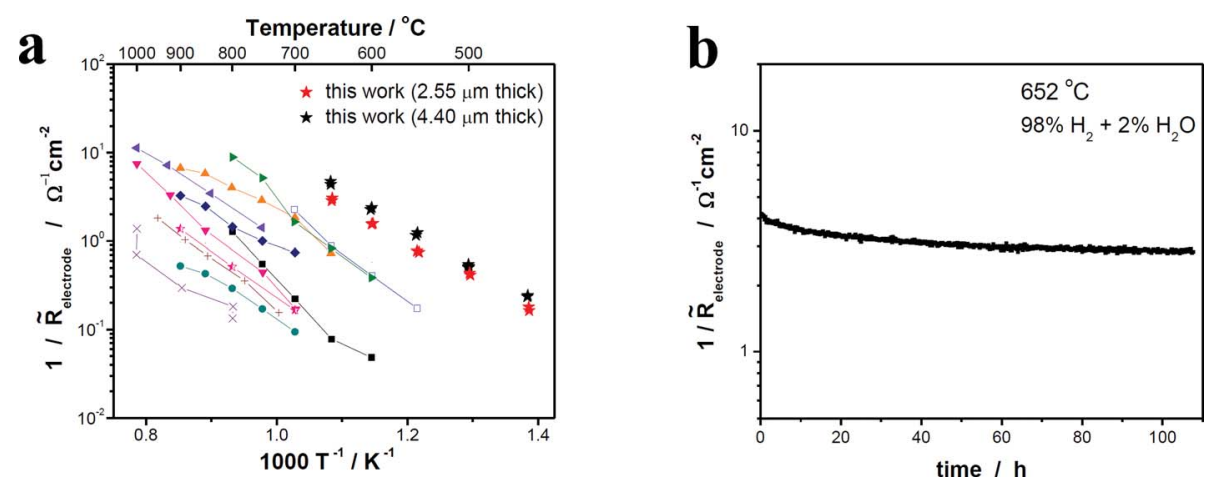

Fig. 7 (a) Arrhenius plot of electrode activity (normalized) for the porous SDC thin film in this study. For comparison, the reported electrode activity (normalized) values for typical anodes are also inserted. Ref. $23(\boldsymbol{\square})=$ slurry-coated $\mathrm{Ni},(\square)=$ slurry-coated $\mathrm{Ni} / \mathrm{Fe}$; ref. $24(\boldsymbol{\Delta})=\mathrm{Pd}$ impregnated Ni/ GDC; ref. $25(\bullet)=$ spin-coated Ni/SDC; ref. $26(\boldsymbol{\nabla})=$ sprayed GDC, $(\triangleleft)=$ Ni impregnated GDC; ref. $27(\bullet)=$ slurry-coated Ni, $(\bullet)=$ GDC impregnated Ni; ref. 28 (文 half-filled) = slurry-coated GDC; ref. $29(+)=$ slurry-coated Ni/YSZ; ref. $30(\times)=$ slurry-coated Ni/YSZ. (b) Electrode activity (normalized) for the porous SDC thin film $\left(4.40 \mu \mathrm{m}\right.$ thick) $v s$. measurement time at $652{ }^{\circ} \mathrm{C}, p \mathrm{H}_{2}=0.98 \mathrm{~atm}$, and $p \mathrm{H}_{2} \mathrm{O}=0.02 \mathrm{~atm}$.

a $2.65 \mu \mathrm{m}$ thick film. The porosity of the films as a function of thickness implied by this approach, applied to all but the thinnest film of Table 1, is summarized in Fig. 6b. The computed porosity falls within the range of 17 to $36 \%$, comparable to the value of 20 to $27 \%$ estimated from the SEM image analysis. In principle, the second term in the capacitance expression can provide information regarding the total SDCgas interfacial area, but the uncertainty in the derived values and lack of detailed information regarding the specific interfacial capacitance precludes such an analysis.

It is of significant technological and scientific relevance to consider what value of electrode performance can be extrapolated or predicted based on measurements of planar, dense films using defined, patterned electrodes. Patterned electrode studies have gained increasing attention as a result of the insight they can provide with respect to mechanistic pathways. ${ }^{3,8,9,16-19}$ Our own previous studies of dense SDC films serve as a useful reference, and a comparative data point is included in Fig. 5a. Here, the dense film result clearly deviates from the trend for the porous films. Indeed, despite the $>300$-fold increase in surface area in the $10.8 \mu \mathrm{m}$ porous film, the increase in activity over that of the planar film is limited to about a factor of 10 . While the precise source of the discrepancy remains unknown, the results raise the tantalizing prospect of achieving a 300 -fold increase in activity in a porous electrode structure in which the specific activity of the dense film is retained (to attain an area-specific electrochemical resistance of less than $0.02 \Omega \mathrm{cm}^{2}$ !) and motivate further study to understand the differences in surface chemistry and structure between planar and porous SDC. In the recent literature, several examples of the impact of PLD growth conditions on the activity of perovskite cathode films for oxygen electroreduction have appeared ${ }^{20-22}$ and may shed light on the behavior of ceria.

Although the observed enhancement in activity falls below that predicted based on an extrapolation of flat film behavior, the increase in surface area achieved here nevertheless creates an SDC electrode with unprecedented overall performance. The results of electrode activity measurements under atmospheres directly relevant to SOFC anodes $\left(98 \% \mathrm{H}_{2}, 2 \% \mathrm{H}_{2} \mathrm{O}\right)$ are presented in Fig. 7 for films 2.55 and $4.40 \mu \mathrm{m}$ in thickness. The temperature dependence, Fig. $7 \mathrm{a}$, is presented in comparison with relevant literature values. The improved activity afforded by the porous ceria electrodes, even when only $2.55 \mu \mathrm{m}$ thick, is striking. Furthermore, the stability as measured for over $100 \mathrm{~h}$, is remarkable given the fine scale of the microstructural features. It is to be emphasized that high activity is attained here in the absence of any metal catalysts. Such high activity points to the significance of ceria nanostructuring as a route to enhanced activity, consistent with recent preliminary findings from the authors' laboratory. ${ }^{3}$

\section{Summary}

Films of $\mathrm{Sm}_{0.2} \mathrm{Ce}_{0.8} \mathrm{O}_{1.9-\delta}$ with highly porous and vertically oriented morphology were fabricated by means of PLD and were explored both as high-performance anodes for SOFCs and as a model system for evaluating the impact of electrode architecture on the electrocatalytic activity. The porous, columnar morphology demonstrates superior performance as a SOFC anode for hydrogen electro-oxidation as compared to typical thin or thick film anodes reported in literature. Furthermore, the structure enables a significant simplification of the complex electrochemical reaction pathways relative to typical cermet electrodes. The activity, defined as the inverse of the interfacial resistance normalized to the electrolyte area, depends almost linearly on the electrode specific surface area (or surface area enhancement factor), reflective of a surface reaction limited process. Films as thick as $10.8 \mu \mathrm{m}$ were found to remain below the thickness of the electrochemical reaction zone, with no indication of the onset of bulk transport limitations. The results suggest a generic approach to high activity electrodes for SOFCs - nanostructuring of mixed ion and electron conductors that inherently display sufficient surface catalytic activity and thermal stability.

\section{Acknowledgements}

This work was supported in part by the Stanford Global Climate Energy Program and by the National Science Foundation under contract number DMR 08-43934. Additional support was provided by the National Science Foundation through the Caltech Center for the Science and Engineering of Materials, a Materials 
Research Science and Engineering Center (DMR-052056). FIBSEM measurements (e.g. Fig. 1e) were kindly performed by Scott Cronin and Scott Barnett of Northwestern University, under NSFDMR support through the Ceramics program.

\section{Notes and references}

1 S. M. Haile, Acta Mater., 2003, 51, 5981-6000.

2 S. P. Jiang and S. H. Chan, J. Mater. Sci., 2004, 39, 4405-4439.

3 W. C. Chueh, Y. Hao, W. Jung and S. M. Haile, Nat. Mater., 2012, 11, 155-161.

4 C. Lu, W. L. Worrell, J. M. Vohs and R. J. Gorte, J. Electrochem. Soc., 2003, 150, A1357-A1359.

5 W. C. Chueh, W. Lai and S. M. Haile, Solid State Ionics, 2008, 179, 1036-1041.

6 T. Nakamura, K. Yashiro, A. Kaimai, T. Otake, K. Sato, T. Kawada and J. Mizusaki, J. Electrochem. Soc., 2008, 155, B1244-B1250.

7 C. Zhang, M. E. Grass, A. H. McDaniel, S. C. DeCaluwe, F. E. Gabaly, Z. Liu, K. F. McCarty, R. L. Farrow, M. A. Linne, Z. Hussain, G. S. Jackson, H. Bluhm and B. W. Eichhorn, Nat. Mater., 2010, 9, 944-949.

8 J. Mizusaki, H. Tagawa, T. Saito, K. Kamitani, T. Yamamura, K. Hirano, S. Ehara, T. Takagi, T. Hikita, M. Ippommatsu, S. Nakagawa and K. Hashimoto, J. Electrochem. Soc., 1994, 141, 2129-2134

9 A. Bieberle, L. P. Meier and L. J. Gauckler, J. Electrochem. Soc., 2001, 148, A646-A656.

10 J. R. Wilson, W. Kobsiriphat, R. Mendoza, H.-Y. Chen, J. M. Hiller, D. J. Miller, K. Thornton, P. W. Voorhees, S. B. Adler and S. A. Barnett, Nat. Mater., 2006, 5, 541-544.

11 W. C. Chueh and S. M. Haile, Phys. Chem. Chem. Phys., 2009, 11, 8144-8148.

12 L. Holzer, B. Münch, B. Iwanschitz, M. Cantoni, T. Hocker and T. Graule, J. Power Sources, 2011, 196, 7076-7089.
13 P. S. Manning, J. D. Sirman, R. A. DeSouza and J. A. Kilner, Solid State Ionics, 1997, 100, 1-10.

14 W. Lai and S. M. Haile, J. Am. Ceram. Soc., 2005, 88, 2979-2997.

15 S. B. Adler, J. A. Lane and B. C. H. Steele, J. Electrochem. Soc., 1996, 143, 3554-3564.

16 F. S. Baumann, J. Fleig, H. U. Habermeier and J. Maier, Solid State Ionics, 2006, 177, 1071-1081.

17 J. L. Hertz, A. Rothschild and H. L. Tuller, J. Electroceram., 2009, 22, 428-435.

18 M. W. Louie and S. M. Haile, Energy Environ. Sci., 2011, 4, 42304238.

19 W. Jung and H. L. Tuller, J. Electrochem. Soc., 2008, 155, B1194B1201.

20 M. Sase, J. Suzuki, K. Yashiro, T. Otake, A. Kaimai, T. Kawada, J. Mizusaki and H. Yugami, Solid State Ionics, 2006, 177, 19611964.

21 J. Januschewsky, M. Ahrens, A. Opitz, F. Kubel and J. Fleig, Adv. Funct. Mater., 2009, 19, 3151-3156.

22 M. Kubicek, A. Limbeck, T. Fromling, H. Hutter and J. Fleig, J. Electrochem. Soc., 2011, 158, B727-B734.

23 T. Ishihara and H. Zhong, Scr. Mater., 2011, 65, 108-111.

24 A. Babaei, S. P. Jiang and J. Li, J. Electrochem. Soc., 2009, 156, B1022-B1029.

25 M. Chen, B. H. Kim, Q. Xu and B. G. Ahn, J. Membr. Sci., 2009, 334, $138-147$

26 S. Primdahl and Y. L. Liu, J. Electrochem. Soc., 2002, 149, A1466A1472.

27 S. P. Jiang, S. Zhang, Y. D. Zhen and A. P. Koh, Electrochem. SolidState Lett., 2004, 7, A282-A285.

28 T. Nakamura, T. Kobayashi, K. Yashiro, A. Kaimai, T. Otake, K. Sato, J. Mizusaki and T. Kawada, J. Electrochem. Soc., 2008, 155, B563-B569.

29 X. G. Wang, N. Nakagawa and K. Kato, J. Electrochem. Soc., 2001, 148, A565-A569.

30 P. Holtappels, I. C. Vinke, L. G. J. de Haart and U. Stimming, J. Electrochem. Soc., 1999, 146, 2976-2982. 